\title{
Existence of Mild Solutions to Fractional Integrodifferential Equations of Neutral Type with Infinite Delay
}

\author{
Fang $\mathrm{Li}^{1}$ and Jun Zhang ${ }^{2}$ \\ ${ }^{1}$ School of Mathematics, Yunnan Normal University, Kunming 650092, China \\ ${ }^{2}$ Department of Mathematics, Central China Normal University, Wuhan 430079, China
}

Correspondence should be addressed to Fang Li, fangli860@gmail.com

Received 5 December 2010; Accepted 30 January 2011

Academic Editor: Jin Liang

Copyright (C) 2011 F. Li and J. Zhang. This is an open access article distributed under the Creative Commons Attribution License, which permits unrestricted use, distribution, and reproduction in any medium, provided the original work is properly cited.

We study the solvability of the fractional integrodifferential equations of neutral type with infinite delay in a Banach space $X$. An existence result of mild solutions to such problems is obtained under the conditions in respect of Kuratowski's measure of noncompactness. As an application of the abstract result, we show the existence of solutions for an integrodifferential equation.

\section{Introduction}

The fractional differential equations are valuable tools in the modeling of many phenomena in various fields of science and engineering; so, they attracted many researchers (cf., e.g., [1-6] and references therein). On the other hand, the integrodifferential equations arise in various applications such as viscoelasticity, heat equations, and many other physical phenomena (cf., e.g., [7-10] and references therein). Moreover, the Cauchy problem for various delay equations in Banach spaces has been receiving more and more attention during the past decades (cf., e.g., [7, 10-15] and references therein).

Neutral functional differential equations arise in many areas of applied mathematics and for this reason, the study of this type of equations has received great attention in the last few years (cf., e.g., [12, 14-16] and references therein). In [12, 16], Hernández and Henríquez studied neutral functional differential equations with infinite delay. In the following, we will extend such results to fractional-order functional differential equations of neutral type with infinite delay. To the authors' knowledge, few papers can be found in the literature for 
the solvability of the fractional-order functional integrodifferential equations of neutral type with infinite delay.

In the present paper, we will consider the following fractional integrodifferential equation of neutral type with infinite delay in Banach space $X$ :

$$
\begin{gathered}
\frac{d^{q}}{d t^{q}}\left(x(t)-h\left(t, x_{t}\right)\right)=A\left(x(t)-h\left(t, x_{t}\right)\right)+\int_{0}^{t} \beta(t, s) f\left(s, x(s), x_{s}\right) d s, \quad t \in[0, T] \\
x(t)=\phi(t) \in D, \quad t \in(-\infty, 0]
\end{gathered}
$$

where $T>0,0<q<1, P$ is a phase space that will be defined later (see Definition 2.5). $A$ is a generator of an analytic semigroup $\{S(t)\}_{t>0}$ of uniformly bounded linear operators on $X$. Then, there exists $M \geq 1$ such that $\|S(t)\| \leq M . h:[0, T] \times D \rightarrow X, f:[0, T] \times X \times D \rightarrow X$, $\beta: D \rightarrow \mathbf{R}(D=\{(t, s) \in[0, T] \times[0, T]: t \geq s\})$, and $x_{t}:(-\infty, 0] \rightarrow X$ defined by $x_{t}(\theta)=x(t+\theta)$, for $\theta \in(-\infty, 0], \phi$ belongs to $D$ and $\phi(0)=0$. The fractional derivative is understood here in the Caputo sense.

The aim of our paper is to study the solvability of (1.1) and present the existence of mild solution of (1.1) based on Kuratowski's measures of noncompactness. Moreover, an example is presented to show an application of the abstract results.

\section{Preliminaries}

Throughout this paper, we set $J:=[0, T]$ and denote by $X$ a real Banach space, by $L(X)$ the Banach space of all linear and bounded operators on $X$, and by $C(J, X)$ the Banach space of all $X$-valued continuous functions on $J$ with the uniform norm topology.

Let us recall the definition of Kuratowski's measure of noncompactness.

Definition 2.1. Let $B$ be a bounded subset of a seminormed linear space $Y$. Kuratowski's measure of noncompactness of $B$ is defined as

$$
\alpha(B)=\inf \{d>0: B \text { has a finite cover by sets of diameter } \leq d\} .
$$

This measure of noncompactness satisfies some important properties.

Lemma 2.2 (see [17]). Let $A$ and $B$ be bounded subsets of $X$. Then,

(1) $\alpha(A) \leq \alpha(B)$ if $A \subseteq B$,

(2) $\alpha(A)=\alpha(\bar{A})$, where $\bar{A}$ denotes the closure of $A$,

(3) $\alpha(A)=0$ if and only if $A$ is precompact,

(4) $\alpha(\lambda A)=|\lambda| \alpha(A), \lambda \in \mathbf{R}$,

(5) $\alpha(A \cup B)=\max \{\alpha(A), \alpha(B)\}$,

(6) $\alpha(A+B) \leq \alpha(A)+\alpha(B)$, where $A+B=\{x+y: x \in A, y \in B\}$,

(7) $\alpha(A+a)=\alpha(A)$ for any $a \in X$,

(8) $\alpha(\overline{\text { conv }} A)=\alpha(A)$, where $\overline{\text { conv }} A$ is the closed convex hull of $A$. 
For $H \subset C(J, X)$, we define

$$
\int_{0}^{t} H(s) d s=\left\{\int_{0}^{t} u(s) d s: u \in H\right\} \quad \text { for } t \in J,
$$

where $H(s)=\{u(s) \in X: u \in H\}$.

The following lemmas will be needed.

Lemma 2.3 (see [17]). If $H \subset C(J, X)$ is a bounded, equicontinuous set, then

$$
\alpha(H)=\sup _{t \in J} \alpha(H(t)) .
$$

Lemma 2.4 (see [18]). If $\left\{u_{n}\right\}_{n=1}^{\infty} \subset L^{1}(J, X)$ and there exists an $m \in L^{1}\left(J, \mathbf{R}^{+}\right)$such that $\left\|u_{n}(t)\right\| \leq$ $m(t)$, a.e. $t \in J$, then $\alpha\left(\left\{u_{n}(t)\right\}_{n=1}^{\infty}\right)$ is integrable and

$$
\alpha\left(\left\{\int_{0}^{t} u_{n}(s) d s\right\}_{n=1}^{\infty}\right) \leq 2 \int_{0}^{t} \alpha\left(\left\{u_{n}(s)\right\}_{n=1}^{\infty}\right) d s .
$$

The following definition about the phase space is due to Hale and Kato [11].

Definition 2.5. A linear space $p$ consisting of functions from $\mathbf{R}^{-}$into $X$ with semi-norm $\|\cdot\|_{p}$ is called an admissible phase space if $D$ has the following properties.

(1) If $x:(-\infty, T] \rightarrow X$ is continuous on $J$ and $x_{0} \in D$, then $x_{t} \in P$ and $x_{t}$ is continuous in $t \in J$ and

$$
\|x(t)\| \leq C\left\|x_{t}\right\|_{p}
$$

where $C \geq 0$ is a constant.

(2) There exist a continuous function $C_{1}(t)>0$ and a locally bounded function $C_{2}(t) \geq 0$ in $t \geq 0$ such that

$$
\left\|x_{t}\right\|_{p} \leq C_{1}(t) \sup _{s \in[0, t]}\|x(s)\|+C_{2}(t)\left\|x_{0}\right\|_{p}
$$

for $t \in[0, T]$ and $x$ as in (1).

(3) The space $P$ is complete.

Remark 2.6. (2.5) in (1) is equivalent to $\|\phi(0)\| \leq C\|\phi\|_{p}$, for all $\phi \in D$.

The following result will be used later.

Lemma 2.7 (see $[19,20])$. Let $U$ be a bounded, closed, and convex subset of a Banach space X such that $0 \in U$, and let $N$ be a continuous mapping of $U$ into itself. If the implication

$$
V=\overline{\operatorname{Conv}} N(V) \text { or } V=N(V) \cup\{0\} \Longrightarrow \alpha(V)=0
$$

holds for every subset $V$ of $U$, then $N$ has a fixed point. 
Let $\Omega$ be a set defined by

$$
\Omega=\left\{x:(-\infty, T] \longrightarrow X \text { such that }\left.x\right|_{(-\infty, 0]} \in D,\left.x\right|_{J} \in C(J, X)\right\} .
$$

Motivated by $[4,5,21]$, we give the following definition of mild solution of (1.1).

Definition 2.8. A function $x \in \Omega$ satisfying the equation

$$
x(t)= \begin{cases}\phi(t), & t \in(-\infty, 0], \\ -Q(t) h(0, \phi)+h\left(t, x_{t}\right)+\int_{0}^{t} \int_{0}^{s} R(t-s) \beta(s, \tau) f\left(\tau, x(\tau), x_{\tau}\right) d \tau d s, & t \in J\end{cases}
$$

is called a mild solution of (1.1), where

$$
\begin{aligned}
& Q(t)=\int_{0}^{\infty} \xi_{q}(\sigma) S\left(t^{q} \sigma\right) d \sigma \\
& R(t)=q \int_{0}^{\infty} \sigma t^{q-1} \xi_{q}(\sigma) S\left(t^{q} \sigma\right) d \sigma
\end{aligned}
$$

and $\xi_{q}$ is a probability density function defined on $(0, \infty)$ such that

$$
\xi_{q}(\sigma)=\frac{1}{q} \sigma^{-1-(1 / q)} \varpi_{q}\left(\sigma^{-1 / q}\right) \geq 0
$$

where

$$
\varpi_{q}(\sigma)=\frac{1}{\pi} \sum_{n=1}^{\infty}(-1)^{n-1} \sigma^{-q n-1} \frac{\Gamma(n q+1)}{n !} \sin (n \pi q), \quad \sigma \in(0, \infty) .
$$

Remark 2.9. According to [22], direct calculation gives that

$$
\|R(t)\| \leq C_{q, M} t^{q-1}, \quad t>0
$$

where $C_{q, M}=q M / \Gamma(1+q)$.

We list the following basic assumptions of this paper.

(H1) $f: J \times X \times p \rightarrow X$ satisfies $f(\cdot, v, w): J \rightarrow X$ is measurable, for all $(v, w) \in X \times p$ and $f(t, \cdot, \cdot): X \times D \rightarrow X$ is continuous for a.e. $t \in J$, and there exist two positive functions $\mu_{i}(\cdot) \in L^{1}\left(J, \mathbf{R}^{+}\right)(i=1,2)$ such that

$$
\|f(t, v, w)\| \leq \mu_{1}(t)\|v\|+\mu_{2}(t)\|w\|_{p}, \quad(t, v, w) \in J \times X \times p .
$$


(H2) For any bounded sets $D_{1} \subset X, D_{2} \subset D$, and $0 \leq s \leq t \leq T$, there exists an integrable positive function $\eta$ such that

$$
\alpha\left(R(t-s) f\left(\tau, D_{1}, D_{2}\right)\right) \leq \eta_{t}(s, \tau)\left(\alpha\left(D_{1}\right)+\sup _{-\infty<\theta \leq 0} \alpha\left(D_{2}(\theta)\right)\right)
$$

where $\eta_{t}(s, \tau):=\eta(t, s, \tau)$ and $\sup _{t \in J} \int_{0}^{t} \int_{0}^{s} \eta_{t}(s, \tau) d \tau d s:=\eta^{*}<\infty$.

(H3) There exists a constant $L>0$ such that

$$
\left\|h\left(t_{1}, \varphi\right)-h\left(t_{2}, \tilde{\varphi}\right)\right\| \leq L\left(\left|t_{1}-t_{2}\right|+\|\varphi-\tilde{\varphi}\|_{p}\right), \quad t_{1}, t_{2} \in J, \varphi, \tilde{\varphi} \in \mathcal{D} .
$$

(H4) For each $t \in J, \beta(t, s)$ is measurable on $[0, t]$ and $\beta(t)=\operatorname{ess} \sup \{|\beta(t, s)|, 0 \leq s \leq t\}$ is bounded on $J$. The map $t \rightarrow B_{t}$ is continuous from $J$ to $L^{\infty}(J, \mathbf{R})$, here, $B_{t}(s)=$ $\beta(t, s)$.

(H5) There exists $M^{*} \in(0,1)$ such that

$$
L C_{1}^{*}+\frac{T^{q} \beta C_{q, M}}{q}\left(\left\|\mu_{1}\right\|_{L^{1}\left(J, \mathbf{R}^{+}\right)}+C_{1}^{*}\left\|\mu_{2}\right\|_{L^{1}\left(J, \mathbf{R}^{+}\right)}\right)<M^{*},
$$

where $C_{1}^{*}=\sup _{t \in J} C_{1}(t), \beta=\sup _{t \in J} \beta(t)$.

\section{Main Result}

In this section, we will apply Lemma 2.7 to show the existence of mild solution of (1.1). To this end, we consider the operator $\Phi: \Omega \rightarrow \Omega$ defined by

$$
(\Phi x)(t)= \begin{cases}\phi(t), & t \in(-\infty, 0] \\ -Q(t) h(0, \phi)+h\left(t, x_{t}\right)+\int_{0}^{t} \int_{0}^{s} R(t-s) \beta(s, \tau) f\left(\tau, x(\tau), x_{\tau}\right) d \tau d s, & t \in J .\end{cases}
$$

It follows from (H1), (H3), and (H4) that $\Phi$ is well defined.

It will be shown that $\Phi$ has a fixed point, and this fixed point is then a mild solution of (1.1).

Let $\bar{y}(\cdot):(-\infty, T] \rightarrow X$ be the function defined by

$$
\bar{y}(t)= \begin{cases}\phi(t), & t \in(-\infty, 0] \\ 0, & t \in J\end{cases}
$$

Set $x(t)=\bar{y}(t)+z(t), t \in(-\infty, T]$. 
It is clear to see that $x$ satisfies (2.9) if and only if $z$ satisfies $z_{0}=0$ and for $t \in J$,

$$
z(t)=-Q(t) h(0, \phi)+h\left(t, \bar{y}_{t}+z_{t}\right)+\int_{0}^{t} \int_{0}^{s} R(t-s) \beta(s, \tau) f\left(\tau, \bar{y}(\tau)+z(\tau), \bar{y}_{\tau}+z_{\tau}\right) d \tau d s
$$

Let $Z_{0}=\left\{z \in \Omega: z_{0}=0\right\}$. For any $z \in Z_{0}$,

$$
\|z\|_{Z_{0}}=\sup _{t \in J}\|z(t)\|+\left\|z_{0}\right\|_{p}=\sup _{t \in J}\|z(t)\| .
$$

Thus, $\left(Z_{0},\|\cdot\|_{Z_{0}}\right)$ is a Banach space. Set

$$
B_{r}=\left\{z \in Z_{0}:\|z\|_{Z_{0}} \leq r\right\}, \quad \text { for some } r>0 .
$$

Then, for $z \in B_{r}$, from(2.6), we have

$$
\begin{aligned}
\left\|\bar{y}_{t}+z_{t}\right\|_{p} & \leq\left\|\bar{y}_{t}\right\|_{p}+\left\|z_{t}\right\|_{p} \\
& \leq C_{1}(t) \sup _{0 \leq \tau \leq t}\|\bar{y}(\tau)\|+C_{2}(t)\left\|\bar{y}_{0}\right\|_{p}+C_{1}(t) \sup _{0 \leq \tau \leq t}\|z(\tau)\|+C_{2}(t)\left\|z_{0}\right\|_{p} \\
& =C_{2}(t)\|\phi\|_{p}+C_{1}(t) \sup _{0 \leq \tau \leq t}\|z(\tau)\| \\
& \leq C_{2}^{*} \cdot\|\phi\|_{p}+C_{1}^{*} r:=r^{*},
\end{aligned}
$$

where $C_{2}^{*}=\sup _{0 \leq \eta \leq T} C_{2}(\eta)$.

In order to apply Lemma 2.7 to show that $\Phi$ has a fixed point, we let $\widetilde{\Phi}: Z_{0} \rightarrow Z_{0}$ be an operator defined by $(\tilde{\Phi} z)(t)=0, t \in(-\infty, 0]$ and for $t \in J$,

$$
\begin{aligned}
(\widetilde{\Phi} z)(t)= & -Q(t) h(0, \phi)+h\left(t, \bar{y}_{t}+z_{t}\right) \\
& +\int_{0}^{t} \int_{0}^{s} R(t-s) \beta(s, \tau) f\left(\tau, \bar{y}(\tau)+z(\tau), \bar{y}_{\tau}+z_{\tau}\right) d \tau d s .
\end{aligned}
$$

Clearly, the operator $\Phi$ has a fixed point is equivalent to $\widetilde{\Phi}$ has one. So, it turns out to prove that $\widetilde{\Phi}$ has a fixed point.

Now, we present and prove our main result.

Theorem 3.1. Assume that (H1)-(H5) are satisfied, then there exists a mild solution of (1.1) on $(-\infty, T]$ provided that $L+16 \beta \eta^{*}<1$.

Proof. For $z \in B_{r}, t \in J$, from (3.6), we have

$$
\begin{aligned}
\left\|f\left(t, \bar{y}(t)+z(t), \bar{y}_{t}+z_{t}\right)\right\| & \leq \mu_{1}(t)\|\bar{y}(t)+z(t)\|+\mu_{2}(t)\left\|\bar{y}_{t}+z_{t}\right\|_{p} \\
& \leq \mu_{1}(t) r+\mu_{2}(t) r^{*} .
\end{aligned}
$$


In view of $(\mathrm{H} 3)$,

$$
\begin{aligned}
\left\|h\left(t, \bar{y}_{t}+z_{t}\right)\right\| & \leq\left\|h\left(t, \bar{y}_{t}+z_{t}\right)-h(t, 0)\right\|+\|h(t, 0)\| \\
& \leq L\left\|\bar{y}_{t}+z_{t}\right\|_{p}+M_{1} \\
& \leq L r^{*}+M_{1}
\end{aligned}
$$

where $M_{1}=\sup _{t \in J}\|h(t, 0)\|$.

Next, we show that there exists some $r>0$ such that $\widetilde{\Phi}\left(B_{r}\right) \subset B_{r}$. If this is not true, then for each positive number $r$, there exist a function $z^{r}(\cdot) \in B_{r}$ and some $t \in J$ such that $\left\|\left(\tilde{\Phi} z^{r}\right)(t)\right\|>r$. However, on the other hand, we have from (3.8), (3.9), and (H4)

$$
\begin{aligned}
r< & \left\|\left(\tilde{\Phi} z^{r}\right)(t)\right\| \\
\leq & \|-Q(t) h(0, \phi)\|+\left\|h\left(t, \bar{y}_{t}+z_{t}^{r}\right)\right\| \\
& +\int_{0}^{t} \int_{0}^{s}\left\|R(t-s) \beta(s, \tau) f\left(\tau, \bar{y}(\tau)+z^{r}(\tau), \bar{y}_{\tau}+z_{\tau}^{r}\right)\right\| d \tau d s \\
\leq & L M\|\phi\|_{p}+M M_{1}+L r^{*}+M_{1}+\beta C_{q, M} \int_{0}^{t} \int_{0}^{s}(t-s)^{q-1}\left[\mu_{1}(\tau) r+\mu_{2}(\tau) r^{*}\right] d \tau d s \\
\leq & L M\|\phi\|_{p}+M M_{1}+L r^{*}+M_{1}+\beta r C_{q, M} \int_{0}^{t} \int_{0}^{s}(t-s)^{q-1} \mu_{1}(\tau) d \tau d s \\
& +\beta r^{*} C_{q, M} \int_{0}^{t} \int_{0}^{s}(t-s)^{q-1} \mu_{2}(\tau) d \tau d s \\
\leq & L\left(M\|\phi\|_{p}+r^{*}\right)+M_{1}(M+1)+\frac{T^{q} \beta C_{q, M}}{q}\left[r\left\|\mu_{1}\right\|_{L^{1}\left(J, \mathbf{R}^{+}\right)}+r^{*}\left\|\mu_{2}\right\|_{L^{1}\left(J, \mathbf{R}^{+}\right)}\right] .
\end{aligned}
$$

Dividing both sides of (3.10) by $r$, and taking $r \rightarrow \infty$, we have

$$
L C_{1}^{*}+\frac{T^{q} \beta C_{q, M}}{q}\left(\left\|\mu_{1}\right\|_{L^{1}\left(J, \mathbf{R}^{+}\right)}+C_{1}^{*}\left\|\mu_{2}\right\|_{L^{1}\left(J, \mathbf{R}^{+}\right)}\right) \geq 1
$$

This contradicts (2.17). Hence, for some positive number $r, \widetilde{\Phi}\left(B_{r}\right) \subset B_{r}$.

Let $\left\{z^{k}\right\}_{k \in \mathbf{N}} \subset B_{r}$ with $z^{k} \rightarrow z$ in $B_{r}$ as $k \rightarrow \infty$. Since $f$ satisfies (H1), for almost every $t \in J$, we get

$$
f\left(t, \bar{y}(t)+z^{k}(t), \bar{y}_{t}+z_{t}^{k}\right) \longrightarrow f\left(t, \bar{y}(t)+z(t), \bar{y}_{t}+z_{t}\right), \quad \text { as } k \rightarrow \infty
$$


In view of (3.6), we have

$$
\left\|\bar{y}_{t}+z_{t}^{k}\right\|_{p} \leq r^{*}
$$

Noting that

$$
\left\|f\left(t, \bar{y}(t)+z^{k}(t), \bar{y}_{t}+z_{t}^{k}\right)-f\left(t, \bar{y}(t)+z(t), \bar{y}_{t}+z_{t}\right)\right\| \leq 2 \mu_{1}(t) r+2 \mu_{2}(t) r^{*},
$$

we have by the Lebesgue Dominated Convergence Theorem that

$$
\begin{aligned}
& \left\|\left(\tilde{\Phi} z^{k}\right)(t)-(\widetilde{\Phi} z)(t)\right\| \\
& \leq\left\|h\left(t, \bar{y}_{t}+z_{t}^{k}\right)-h\left(t, \bar{y}_{t}+z_{t}\right)\right\| \\
& \quad+\int_{0}^{t} \int_{0}^{s}\left\|R(t-s) \beta(s, \tau)\left[f\left(\tau, \bar{y}(\tau)+z^{k}(\tau), \bar{y}_{\tau}+z_{\tau}^{k}\right)-f\left(\tau, \bar{y}(\tau)+z(\tau), \bar{y}_{\tau}+z_{\tau}\right)\right]\right\| d \tau d s \\
& \leq L\left\|z_{t}^{k}-z_{t}\right\|_{p} \\
& \quad+\beta C_{q, M} \int_{0}^{t} \int_{0}^{s}(t-s)^{q-1}\left\|f\left(\tau, \bar{y}(\tau)+z^{k}(\tau), \bar{y}_{\tau}+z_{\tau}^{k}\right)-f\left(\tau, \bar{y}(\tau)+z(\tau), \bar{y}_{\tau}+z_{\tau}\right)\right\| d \tau d s \\
& \longrightarrow 0, \quad k \longrightarrow \infty .
\end{aligned}
$$

Therefore, we obtain

$$
\lim _{k \rightarrow \infty}\left\|\widetilde{\Phi} z^{k}-\tilde{\Phi} z\right\|_{Z_{0}}=0
$$

This shows that $\widetilde{\Phi}$ is continuous.

Set

$$
G\left(\cdot, \bar{y}(\cdot)+z(\cdot), \bar{y}_{(\cdot)}+z_{(\cdot)}\right):=\int_{0} \beta(\cdot, \tau) f\left(\tau, \bar{y}(\tau)+z(\tau), \bar{y}_{\tau}+z_{\tau}\right) d \tau
$$

Let $0<t_{2}<t_{1}<T$ and $z \in B_{r}$, then we can see

$$
\left\|(\widetilde{\Phi} z)\left(t_{1}\right)-(\widetilde{\Phi} z)\left(t_{2}\right)\right\| \leq I_{1}+I_{2}+I_{3}+I_{4}
$$


where

$$
\begin{aligned}
& I_{1}=\left\|Q\left(t_{1}\right)-Q\left(t_{2}\right)\right\| \cdot\|h(0, \phi)\|, \\
& I_{2}=\left\|h\left(t_{1}, \bar{y}_{t_{1}}+z_{t_{1}}\right)-h\left(t_{2}, \bar{y}_{t_{2}}+z_{t_{2}}\right)\right\|, \\
& I_{3}=\left\|\int_{0}^{t_{2}}\left[R\left(t_{1}-s\right)-R\left(t_{2}-s\right)\right] G\left(s, \bar{y}(s)+z(s), \bar{y}_{s}+z_{s}\right) d s\right\|, \\
& I_{4}=\int_{t_{2}}^{t_{1}}\left\|R\left(t_{1}-s\right)\right\|\left\|G\left(s, \bar{y}(s)+z(s), \bar{y}_{s}+z_{s}\right)\right\| d s .
\end{aligned}
$$

It follows the continuity of $S(t)$ in the uniform operator topology for $t>0$ that $I_{1}$ tends to 0 , as $t_{2} \rightarrow t_{1}$. The continuity of $h$ ensures that $I_{2}$ tends to 0 , as $t_{2} \rightarrow t_{1}$.

For $I_{3}$, we have

$$
\begin{aligned}
I_{3} \leq & q \int_{0}^{t_{2}} \int_{0}^{\infty} \sigma\left\|\left[\left(t_{1}-s\right)^{q-1}-\left(t_{2}-s\right)^{q-1}\right] \xi_{q}(\sigma) S\left(\left(t_{1}-s\right)^{q} \sigma\right) G\left(s, \bar{y}(s)+z(s), \bar{y}_{s}+z_{s}\right)\right\| d \sigma d s \\
& +q \int_{0}^{t_{2}} \int_{0}^{\infty} \sigma\left(t_{2}-s\right)^{q-1} \xi_{q}(\sigma)\left\|S\left(\left(t_{1}-s\right)^{q} \sigma\right)-S\left(\left(t_{2}-s\right)^{q} \sigma\right)\right\| \\
& \times\left\|G\left(s, \bar{y}(s)+z(s), \bar{y}_{s}+z_{s}\right)\right\| d \sigma d s \\
\leq & C_{q, M} \int_{0}^{t_{2}}\left|\left(t_{1}-s\right)^{q-1}-\left(t_{2}-s\right)^{q-1}\right|\left\|G\left(s, \bar{y}(s)+z(s), \bar{y}_{s}+z_{s}\right)\right\| d s \\
& +q \int_{0}^{t_{2}} \int_{0}^{\infty} \sigma\left(t_{2}-s\right)^{q-1} \xi_{q}(\sigma)\left\|S\left(\left(t_{1}-s\right)^{q} \sigma\right)-S\left(\left(t_{2}-s\right)^{q} \sigma\right)\right\| \\
\leq & \beta\left[r\left\|\mu_{1}\right\|_{L^{1}\left(J, \mathbf{R}^{+}\right)}+r^{*}\left\|\mu_{2}\right\|_{L^{1}\left(J, \mathbf{R}^{+}\right)}\right] \\
& \times\left[C_{q, M} \int_{0}^{t_{2}}\left|\left(t_{1}-s\right)^{q-1}-\left(t_{2}-s\right)^{q-1}\right| d s\right. \\
& \left.+q \int_{0}^{t_{2}} \int_{0}^{\infty} \sigma\left(t_{2}-s\right)^{q-1} \xi_{q}(\sigma)\left\|S\left(\left(t_{1}-s\right)^{q} \sigma\right)-S\left(\left(t_{2}-s\right)^{q} \sigma\right)\right\| d \sigma d s\right] .
\end{aligned}
$$


Clearly, the first term on the right-hand side of (3.20) tends to 0 as $t_{2} \rightarrow t_{1}$. The second term on the right-hand side of (3.20) tends to 0 as $t_{2} \rightarrow t_{1}$ as a consequence of the continuity of $S(t)$ in the uniform operator topology for $t>0$.

In view of the assumption of $\mu_{i}(s)(i=1,2)$ and $(3.8)$, we see that

$$
\begin{aligned}
I_{4} & \leq C_{q, M} \int_{t_{2}}^{t_{1}}\left(t_{1}-s\right)^{q-1}\left\|G\left(s, \bar{y}(s)+z(s), \bar{y}_{s}+z_{s}\right)\right\| d s \\
& \leq \beta C_{q, M}\left[r\left\|\mu_{1}\right\|_{L^{1}\left(J, \mathbf{R}^{+}\right)}+r^{*}\left\|\mu_{2}\right\|_{L^{1}\left(J, \mathbf{R}^{+}\right)}\right] \int_{t_{2}}^{t_{1}}\left(t_{1}-s\right)^{q-1} d s \\
& \longrightarrow 0, \quad \text { as } t_{2} \longrightarrow t_{1} .
\end{aligned}
$$

Thus, $\widetilde{\Phi}\left(B_{r}\right)$ is equicontinuous.

Now, let $V$ be an arbitrary subset of $B_{r}$ such that $V \subset \overline{\operatorname{conv}}(\tilde{\Phi}(V) \cup\{0\})$.

Set $\left(\widetilde{\Phi}_{1} z\right)(t)=h\left(t, \bar{y}_{t}+z_{t}\right)$,

$$
\left(\widetilde{\Phi}_{2} z\right)(t)=-Q(t) h(0, \phi)+\int_{0}^{t} \int_{0}^{s} R(t-s) \beta(s, \tau) f\left(\tau, \bar{y}(\tau)+z(\tau), \bar{y}_{\tau}+z_{\tau}\right) d \tau d s
$$

Noting that for $z, \tilde{z} \in V$, we have

$$
\left\|h\left(t, \bar{y}_{t}+\tilde{z}_{t}\right)-h\left(t, \bar{y}_{t}+z_{t}\right)\right\| \leq L\left\|\tilde{z}_{t}-z_{t}\right\|_{p}
$$

Thus,

$$
\alpha\left(h\left(t, \bar{y}_{t}+V_{t}\right)\right) \leq L \alpha\left(V_{t}\right) \leq L \sup _{-\infty<\theta \leq 0} \alpha(V(t+\theta))=\operatorname{Lsup}_{0 \leq \tau \leq t} \alpha(V(\tau)) \leq L \alpha(V),
$$

where $V_{t}=\left\{z_{t}: z \in V\right\}$. Therefore, $\alpha\left(\widetilde{\Phi}_{1} V\right)=\sup _{t \in J} \alpha\left(\left(\widetilde{\Phi}_{1} V\right)(t)\right) \leq L \alpha(V)$.

Moreover, for any $\varepsilon>0$ and bounded set $D$, we can take a sequence $\left\{v_{n}\right\}_{n=1}^{\infty} \subset D$ such that $\alpha(D) \leq 2 \alpha\left(\left\{v_{n}\right\}\right)+\varepsilon$ (see [23], P125). Thus, for $\left\{v_{n}\right\}_{n=1}^{\infty} \subset V$, noting that the choice of $V$, and from Lemmas 2.2-2.4 and (H2), we have 


$$
\begin{aligned}
\alpha\left(\widetilde{\Phi}_{2} V\right) & \leq 2 \alpha\left(\left\{\widetilde{\Phi}_{2} v_{n}\right\}\right)+\varepsilon=2 \sup _{t \in J} \alpha\left(\left\{\tilde{\Phi}_{2} v_{n}(t)\right\}\right)+\varepsilon \\
& =2 \sup _{t \in J} \alpha\left(\left\{\int_{0}^{t} R(t-s) \int_{0}^{s} \beta(s, \tau) f\left(\tau, \bar{y}(\tau)+v_{n}(\tau), \bar{y}_{\tau}+v_{n \tau}\right) d \tau d s\right\}\right)+\varepsilon \\
& \leq 4 \sup _{t \in J} \int_{0}^{t} \alpha\left(\left\{R(t-s) \int_{0}^{s} \beta(s, \tau) f\left(\tau, \bar{y}(\tau)+v_{n}(\tau), \bar{y}_{\tau}+v_{n \tau}\right) d \tau\right\}\right) d s+\varepsilon \\
& \leq 8 \sup _{t \in J} \int_{0}^{t} \int_{0}^{s} \alpha\left(\left\{R(t-s) \beta(s, \tau) f\left(\tau, \bar{y}(\tau)+v_{n}(\tau), \bar{y}_{\tau}+v_{n \tau}\right)\right\}\right) d \tau d s+\varepsilon \\
& \leq 8 \beta \sup _{t \in J} \int_{0}^{t} \int_{0}^{s} \alpha\left(\left\{R(t-s) f\left(\tau, \bar{y}(\tau)+v_{n}(\tau), \bar{y}_{\tau}+v_{n \tau}\right)\right\}\right) d \tau d s+\varepsilon \\
& \leq 8 \beta \sup _{t \in J} \int_{0}^{t} \int_{0}^{s} \eta_{t}(s, \tau)\left[\alpha\left(\left\{v_{n}(\tau)\right\}\right)+\sup _{-\infty<\theta \leq 0} \alpha\left(\left\{v_{n}(\theta+\tau)\right\}\right)\right] d \tau d s+\varepsilon \\
& \leq 8 \beta \sup _{t \in J} \int_{0}^{t} \int_{0}^{s} \eta_{t}(s, \tau)\left[\alpha\left(\left\{v_{n}\right\}\right)+\sup _{0 \leq \mu \leq \tau} \alpha\left(\left\{v_{n}(\mu)\right\}\right)\right] d \tau d s+\varepsilon \\
& \leq 16 \beta \alpha\left(\left\{v_{n}\right\}\right) \sup _{t \in J} \int_{0}^{t} \int_{0}^{s} \eta_{t}(s, \tau) d \tau d s+\varepsilon \leq 16 \beta \eta^{*} \alpha(V)+\varepsilon .
\end{aligned}
$$

It follows from Lemma 2.2 that

$$
\alpha(V) \leq \alpha(\widetilde{\Phi} V) \leq \alpha\left(\widetilde{\Phi}_{1} V\right)+\alpha\left(\widetilde{\Phi}_{2} V\right) \leq\left(L+16 \beta \eta^{*}\right) \alpha(V)+\varepsilon,
$$

since $\varepsilon$ is arbitrary, we can obtain

$$
\alpha(V) \leq\left(L+16 \beta \eta^{*}\right) \alpha(V) .
$$

Hence, $\alpha(V)=0$. Applying now Lemma 2.7, we conclude that $\widetilde{\Phi}$ has a fixed point $z^{*}$ in $B_{r}$. Let $x(t)=\bar{y}(t)+z^{*}(t), t \in(-\infty, T]$, then $x(t)$ is a fixed point of the operator $\Phi$ which is a mild solution of (1.1). 


\section{Application}

In this section, we consider the following integrodifferential model:

$$
\begin{aligned}
& \frac{\partial^{q}}{\partial t^{q}}\left[v(t, \xi)-t \int_{-\infty}^{0} \gamma_{1}(\theta) \frac{|v(t+\theta, \xi)|}{1+|v(t+\theta, \xi)|} d \theta\right] \\
& =\frac{\partial^{2}}{\partial \xi^{2}}\left[v(t, \xi)-t \int_{-\infty}^{0} \gamma_{1}(\theta) \frac{|v(t+\theta, \xi)|}{1+|v(t+\theta, \xi)|} d \theta\right] \\
& \quad+\int_{0}^{t}(t-s) \frac{s^{k}}{k} \sin |v(s, \xi)| \cdot \int_{0}^{s} \cos v(\tau, \xi) d \tau d s \\
& \quad+\int_{0}^{t}(t-s) \int_{-\infty}^{0} \gamma_{2}(\theta) \sin \left(s^{2}|v(s+\theta, \xi)|\right) d \theta d s, \\
& v(t, 0)-t \int_{-\infty}^{0} \gamma_{1}(\theta) \frac{|v(t+\theta, 0)|}{1+|v(t+\theta, 0)|} d \theta=0, \\
& v(t, 1)-t \int_{-\infty}^{0} \gamma_{1}(\theta) \frac{|v(t+\theta, 1)|}{1+|v(t+\theta, 1)|} d \theta=0, \\
& v(\theta, \xi)=v_{0}(\theta, \xi), \quad-\infty<\theta \leq 0,
\end{aligned}
$$

where $0 \leq t \leq 1, \xi \in[0,1], k \in \mathbf{N}, \gamma_{1}, \gamma_{2}:(-\infty, 0] \rightarrow \mathbf{R}, v_{0}:(-\infty, 0] \times[0,1] \rightarrow \mathbf{R}$ are continuous functions, and $\int_{-\infty}^{0}\left|\gamma_{i}(\theta)\right| d \theta<\infty(i=1,2)$.

Set $X=L^{2}([0,1], \mathbf{R})$ and define $A$ by

$$
\begin{gathered}
D(A)=H^{2}(0,1) \cap H_{0}^{1}(0,1), \\
A u=u^{\prime \prime} .
\end{gathered}
$$

Then, A generates a compact, analytic semigroup $S(\cdot)$ of uniformly bounded, linear operators, and $\|S(t)\| \leq 1$.

Let the phase space $P$ be $B U C\left(\mathbf{R}^{-}, X\right)$, the space of bounded uniformly continuous functions endowed with the following norm:

$$
\|\varphi\|_{p}=\sup _{-\infty<\theta \leq 0}|\varphi(\theta)|, \quad \forall \varphi \in D
$$

then we can see that $C_{1}(t)=1$ in (2.6). 
For $t \in[0,1], \xi \in[0,1]$ and $\varphi \in B U C\left(\mathbf{R}^{-}, X\right)$, we set

$$
\begin{gathered}
x(t)(\xi)=v(t, \xi), \\
\phi(\theta)(\xi)=v_{0}(\theta, \xi), \quad \theta \in(-\infty, 0], \\
h(t, \varphi)(\xi)=t \int_{-\infty}^{0} \gamma_{1}(\theta) \frac{|\varphi(\theta)(\xi)|}{1+|\varphi(\theta)(\xi)|} d \theta, \\
\beta(t, s)=t-s, \\
f(t, x(t), \varphi)(\xi)=\frac{t^{k}}{k} \sin |x(t)(\xi)| \cdot \int_{0}^{t} \cos x(s)(\xi) d s+\int_{-\infty}^{0} \gamma_{2}(\theta) \sin \left(t^{2}|\varphi(\theta)(\xi)|\right) d \theta .
\end{gathered}
$$

Then (4.1) can be reformulated as the abstract (1.1).

Moreover, for $t \in[0,1]$, we can see

$$
\begin{aligned}
\|f(t, x(t), \varphi)(\xi)\| & \leq \frac{t^{k+1}}{k}\|x(t)\|+t^{2}\|\varphi\|_{p} \int_{-\infty}^{0}\left|\gamma_{2}(\theta)\right| d \theta \\
& =\mu_{1}(t)\|x(t)\|+\mu_{2}(t)\|\varphi\|_{p^{\prime}}
\end{aligned}
$$

where $\mu_{1}(t):=t^{k+1} / k, \mu_{2}(t):=t^{2} \int_{-\infty}^{0}\left|\gamma_{2}(\theta)\right| d \theta$.

For $t_{1}, t_{2} \in[0,1], \varphi, \tilde{\varphi} \in \mathcal{D}$, we have

$$
\begin{aligned}
\left\|h\left(t_{1}, \varphi\right)-h\left(t_{2}, \tilde{\varphi}\right)\right\| \leq & \left|t_{1}-t_{2}\right| \int_{-\infty}^{0}\left\|r_{1}(\theta) \frac{|\varphi(\theta)(\xi)|}{1+|\varphi(\theta)(\xi)|}\right\| d \theta \\
& +t_{2} \int_{-\infty}^{0}\left\|r_{1}(\theta)\left(\frac{|\varphi(\theta)(\xi)|}{1+|\varphi(\theta)(\xi)|}-\frac{|\tilde{\varphi}(\theta)(\xi)|}{1+|\tilde{\varphi}(\theta)(\xi)|}\right)\right\| d \theta \\
\leq & \left|t_{1}-t_{2}\right| \int_{-\infty}^{0}\left|r_{1}(\theta)\right| d \theta+\int_{-\infty}^{0}\left|r_{1}(\theta)\right| d \theta \cdot\|\varphi-\tilde{\varphi}\|_{p} \\
& =L\left(\left|t_{1}-t_{2}\right|+\|\varphi-\tilde{\varphi}\|_{p}\right),
\end{aligned}
$$

where $L=\int_{-\infty}^{0}\left|\gamma_{1}(\theta)\right| d \theta$.

Suppose further that there exists a constant $M^{*} \in(0,1)$ such that

$$
L+\frac{C_{q, M}}{q}\left(\left\|\mu_{1}\right\|_{L^{1}\left([0,1], \mathbf{R}^{+}\right)}+\left\|\mu_{2}\right\|_{L^{1}\left([0,1], \mathbf{R}^{+}\right)}\right)<M^{*}
$$

then (4.1) has a mild solution by Theorem 3.1.

For example, if we put

$$
\gamma_{1}(\theta)=\gamma_{2}(\theta)=e^{k \theta}, \quad q=0.5, \quad k=2,
$$


then $L=1 / 2, C_{q, M}=1 / \Gamma(0.5)=1 / \sqrt{\pi},\left\|\mu_{1}\right\|_{L^{1}\left([0,1], \mathbf{R}^{+}\right)}=1 / 8,\left\|\mu_{2}\right\|_{L^{1}\left([0,1], \mathbf{R}^{+}\right)}=1 / 6$. Thus, we see

$$
L+\frac{C_{q, M}}{q}\left(\left\|\mu_{1}\right\|_{L^{1}\left([0,1], \mathbf{R}^{+}\right)}+\left\|\mu_{2}\right\|_{L^{1}\left([0,1], \mathbf{R}^{+}\right)}\right)=\frac{1}{2}+\frac{2}{\sqrt{\pi}}\left(\frac{1}{8}+\frac{1}{6}\right)<0.9<1
$$

\section{Acknowledgments}

The authors are grateful to the referees for their valuable suggestions. F. Li is supported by the NSF of Yunnan Province (2009ZC054M). J. Zhang is supported by Tianyuan Fund of Mathematics in China (11026100).

\section{References}

[1] R. P. Agarwal, B. de Andrade, and C. Cuevas, "On type of periodicity and ergodicity to a class of fractional order differential equations," Advances in Difference Equations, vol. 2010, Article ID 179750, 25 pages, 2010.

[2] H. M. Ahmed, "Boundary controllability of nonlinear fractional integrodifferential systems," Advances in Difference Equations, vol. 2010, Article ID 279493, 9 pages, 2010.

[3] A. Alsaedi and B. Ahmad, "Existence of solutions for nonlinear fractional integro-differential equations with three-point nonlocal fractional boundary conditions," Advances in Difference Equations, vol. 2010, Article ID 691721, 10 pages, 2010.

[4] M. M. El-Borai, "Some probability densities and fundamental solutions of fractional evolution equations," Chaos, Solitons and Fractals, vol. 14, no. 3, pp. 433-440, 2002.

[5] M. M. El-Borai, "On some stochastic fractional integro-differential equations," Advances in Dynamical Systems and Applications, vol. 1, no. 1, pp. 49-57, 2006.

[6] G. M. Mophou and G. M. N'Guérékata, "Existence of the mild solution for some fractional differential equations with nonlocal conditions," Semigroup Forum, vol. 79, no. 2, pp. 315-322, 2009.

[7] J. Liang, T.-J. Xiao, and J. van Casteren, "A note on semilinear abstract functional differential and integrodifferential equations with infinite delay," Applied Mathematics Letters, vol. 17, no. 4, pp. 473477, 2004.

[8] J. Liang and T.-J. Xiao, "Semilinear integrodifferential equations with nonlocal initial conditions," Computers \& Mathematics with Applications, vol. 47, no. 6-7, pp. 863-875, 2004.

[9] J. Liang, J. H. Liu, and T.-J. Xiao, "Nonlocal problems for integrodifferential equations," Dynamics of Continuous, Discrete E Impulsive Systems. Series A, vol. 15, no. 6, pp. 815-824, 2008.

[10] T.-J. Xiao and J. Liang, "Blow-up and global existence of solutions to integral equations with infinite delay in Banach spaces," Nonlinear Analysis: Theory, Methods \& Applications, vol. 71, no. 12, pp. e1442e1447, 2009.

[11] J. K. Hale and J. Kato, "Phase space for retarded equations with infinite delay," Funkcialaj Ekvacioj, vol. 21, no. 1, pp. 11-41, 1978.

[12] E. Hernández and H. R. Henríquez, "Existence results for partial neutral functional-differential equations with unbounded delay," Journal of Mathematical Analysis and Applications, vol. 221, no. 2, pp. 452-475, 1998.

[13] J. Liang and T. J. Xiao, "Functional-differential equations with infinite delay in Banach spaces," International Journal of Mathematics and Mathematical Sciences, vol. 14, no. 3, pp. 497-508, 1991.

[14] G. M. Mophou and G. M. N'Guérékata, "Existence of mild solutions of some semilinear neutral fractional functional evolution equations with infinite delay," Applied Mathematics and Computation, vol. 216, no. 1, pp. 61-69, 2010.

[15] G. M. Mophou and G. M. N'Guérékata, “A note on a semilinear fractional differential equation of neutral type with infinite delay," Advances in Difference Equations, vol. 2010, Article ID 674630, 8 pages, 2010.

[16] E. Hernández and H. R. Henríquez, "Existence of periodic solutions of partial neutral functionaldifferential equations with unbounded delay," Journal of Mathematical Analysis and Applications, vol. 221, no. 2, pp. 499-522, 1998. 
[17] J. Banaś and K. Goebel, Measures of Noncompactness in Banach Spaces, vol. 60 of Lecture Notes in Pure and Applied Mathematics, Marcel Dekker, New York, NY, USA, 1980.

[18] H.-P. Heinz, "On the behaviour of measures of noncompactness with respect to differentiation and integration of vector-valued functions," Nonlinear Analysis: Theory, Methods \& Applications, vol. 7, no. 12, pp. 1351-1371, 1983.

[19] R. P. Agarwal, M. Meehan, and D. O'Regan, Fixed Point Theory and Applications, vol. 141 of Cambridge Tracts in Mathematics, Cambridge University Press, Cambridge, UK, 2001.

[20] S. Szufla, "On the application of measure of noncompactness to existence theorems," Rendiconti del Seminario Matematico della Università di Padova, vol. 75, pp. 1-14, 1986.

[21] Y. Zhou and F. Jiao, "Nonlocal Cauchy problem for fractional evolution equations," Nonlinear Analysis: Real World Applications, vol. 11, no. 5, pp. 4465-4475, 2010.

[22] F. Mainardi, P. Paradisi, and R. Gorenflo, "Probability distributions generated by fractional diffusion equations," in Econophysics: An Emerging Science, J. Kertesz and I. Kondor, Eds., Kluwer Academic Publishers, Dordrecht, The Netherlands, 2000.

[23] D. Bothe, "Multivalued perturbations of $m$-accretive differential inclusions," Israel Journal of Mathematics, vol. 108, pp. 109-138, 1998. 